\title{
Optical properties of a nanostructured glass-based film using spectroscopic ellipsometry
}

G. E. Jellison, Jr. ${ }^{1}$, T. Aytug ${ }^{2}$, A. R. Lupini ${ }^{3}$, M. P. Paranthaman ${ }^{2}$ and P. C. Joshi ${ }^{3}$

Affiliations:

${ }^{1}$ Oak Ridge National Laboratory

P. O. Box 2008, Mail Stop 6069

Oak Ridge, TN 37831 USA

jellisongejr@ornl.gov

Phone: $\quad 8655767309$

(Corresponding author, retired)

${ }^{2}$ Chemical Sciences Division

Oak Ridge National Laboratory

P. O. Box 2008, Mail Stop 6100

Oak Ridge, TN 37831 USA

aytugt@ornl.gov (Aytug) paranthamanm@ornl.gov (Paranthaman)

Phone $\quad 8655746571 \quad 8655745045$

${ }^{3}$ Materials Science and Technology Division

Oak Ridge National Laboratory

P. O. Box 2008 Mail Stop 6071

Oak Ridge, TN 37831 USA

Arl1000@ornl.gov (Lupini)_joshipc@ornl.gov (Joshi)

Phone: $\quad 8655746281 \quad 8655764718$ 


\begin{abstract}
Nanostructured glass films, which are fabricated using spinodally phase-separated low-alkali glasses, have several interesting and useful characteristics, including being robust, non-wetting and antireflective. Spectroscopic ellipsometry measurements have been performed on one such film and its optical properties were analyzed using a 5-layer structural model of the near-surface region. Since the glass and the film are transparent over the spectral region of the measurement, the Sellmeier model is used to parameterize the dispersion in the refractive index. To simulate the variation of the optical properties of the film over the spot size of the ellipsometer $(\sim 3 \times 5 \mathrm{~mm})$, the Sellmeier amplitude is convoluted using a Gaussian distribution. The transition layers between the ambient and the film and between the film and the substrate are modeled as graded layers, where the refractive index varies as a function of depth. These layers are modeled using a two-component Bruggeman effective medium approximation where the two components are the layer above and the layer below. The fraction is continuous through the transition layer and is modelled using the incomplete beta function.
\end{abstract}

\title{
Keywords
}

Spectroscopic ellipsometry; nanostructured glass films; Bruggeman effective medium approximation; incomplete beta function 


\section{Introduction}

Recently, a unique manufacturing

process has been discovered that results in

the formation of unique nanostructured

porous glass films [1, 2]. Microscopically, the

films consist of a silica material interlaced

with pores (See Figure 1), where the feature

size is $50-500 \mathrm{~nm}$. As a result, the films have

a number of interesting and potentially

useful characteristics. First of all, the films

are structurally hydrophilic, making them

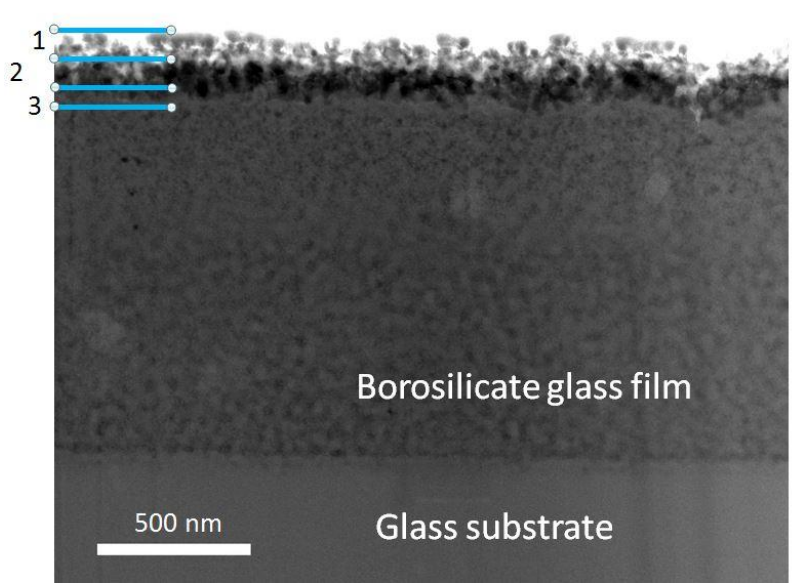

Figure 1. 'Z-contrast' cross-sectional scanning transmission electron microscopy (STEM) image of the glass coating. The fiduciary mark represents $500 \mathrm{~nm}$. The layers marked 1, 2, and 3 represent the transition layer between air and the film, the film, and the transition layer between the film and the unetched borosilicate glass film.

anti-fogging due to capillary action created by the porous film network, enabling condensation to rapidly spread and wet the surface. After appropriate low-surface energy chemical modification, the surface provides superhydrophobicity; that is they actually repel water, making water droplets to ball up with a contact angle greater than $150^{\circ}$. Because of this property, the films can be cleaned very easily, where the dust particles and other debris are incorporated into the water droplets, which then roll off the film surface, taking the dirt with them. These films are an integral part of the underlying substrate, making them robust against abrasions. Since the films consist of glass and voids, they are transparent, and they have a refractive index intermediate between the refractive index of the glass and air, making them anti-reflective. 
The films are created by a process that exploits the spinodal decomposition of low-alkali borosilicate glass at elevated temperatures. For the present case, spinodal decomposition is the rapid phase separation of a glass mixture into two different phases that is not caused by nucleation. The first step involves the deposition of a phase separating sodium borosilicate glass onto a glass substrate of a particular composition using magnetron sputtering. The material is then heated to $500-700{ }^{\circ} \mathrm{C}$ which causes the spinodal decomposition. Typical microstructure of this film is shown in Fig. 1 and is labeled as borosilicate glass film. An etchant is then used that preferentially etches one of the components, leaving behind a combination of voids and glass. For the sample presented in Fig. 1 the etching time was short thus only the top $\sim 200 \mathrm{~nm}$ was affected by the etchant. Note that the structural and physical properties of the film can be modified by a number of process parameters, such as the heating temperature and duration, the composition of the substrate and the glass film, the etchant selected and the duration of etching and its concentration.

A glass sample that has been coated with such a nanostructured film on the front will exhibit increased broadband transmission and reduced reflectivity ( $300-800 \mathrm{~nm})$ [2]. Both the increased transmission and reduced reflectivity do not depend upon the direction of the light beam incident upon the sample. Below $\sim 300 \mathrm{~nm}$, the transmission is reduced, indicating that the film also preferentially scatters in the blue and ultraviolet associated with the small dimensions of the porous silica film features.

This paper is an extension of our previous work [2], where we have utilized a considerably more complicated model for the ellipsometry data analysis. The primary conclusion of 
reference 2 remains intact: The nanotextured silica film has a refractive index between the ambient air and the glass substrate. Moreover, the porosity of the coating is not a single value, but rather changes continuously from the surface to the glass substrate. While the analysis of ref. 2 used 3 layers of different void fractions, the analysis presented in this paper uses the incomplete Beta function to parameterize the void fraction in the film as a function of depth.

This function has a range and domain of 0 to 1 and requires only two fitting parameters to model most reasonable variations in void fraction versus depth. The resulting fit is considerably better than that presented in ref. 2 and reveals the complexity of the void fraction profile at the surface of the glass surface.

\section{Ellipsometry measurements}

Spectroscopic ellipsometry measurements were performed using the two modulator generalized ellipsometer (2-MGE) [3, 4] on a nanostructured glass film and the underlying glass substrate before the film was grown. The 2-MGE measures 8 parameters which are related directly to the sample $4 \mathrm{X} 4$ Muller matrix. If the sample is isotropic, then the off-block diagonal elements of the sample Mueller matrix are zero, and the instrument then measures the quantities $N, S$, and $C$. If the sample does not exhibit depolarization, then $\beta^{2}=N^{2}+S^{2}+C^{2}=1$ and the measured quantities can then be expressed using the traditional ellipsometry parameters:

$$
\rho=\frac{r_{p}}{r_{s}}=\tan (\psi) e^{i \Delta}=\frac{C+i S}{\beta+N}
$$


where $r_{p}$ and $r_{s}$ are the complex reflection coefficients for light polarized parallel and perpendicular to the plane of incidence and $\psi$ and $\Delta$ are the traditional ellipsometry angles. The $\mathrm{N}, \mathrm{S}$, and $\mathrm{C}$ parameters can also be expressed in terms of $\psi$ and $\Delta: \mathrm{N}=\cos (2 \psi), \mathrm{S}=$ $\sin (2 \psi) \sin (\Delta)$, and $C=\sin (2 \psi) \cos (\Delta)$. While any of these parameter sets can be used to analyze the data, it is critical to propagate the errors into the parameter representation used to fit the data. We have used the $\rho$ representation, since it eliminates the discontinuity commonly found in $\Delta$ when $\Delta$ goes from $0^{\circ}$ to $360^{\circ}$.

The $\rho$ data and estimated error are shown in Fig. 2 for the film, along with the best fit to the data, discussed below. Clearly, there is a resonance near $3.5 \mathrm{eV}$, but the error in both $\operatorname{Re}(\rho)$ and $\operatorname{Im}(\rho)$ is largest there as well. The error in the wings of the resonance is considerably less, meaning that the fitting procedure must weight this region of the spectrum more than the region near the resonance. Note that the wings of the data show an oscillation which is considerably larger than the error; any realistic model of the data must take this into account.
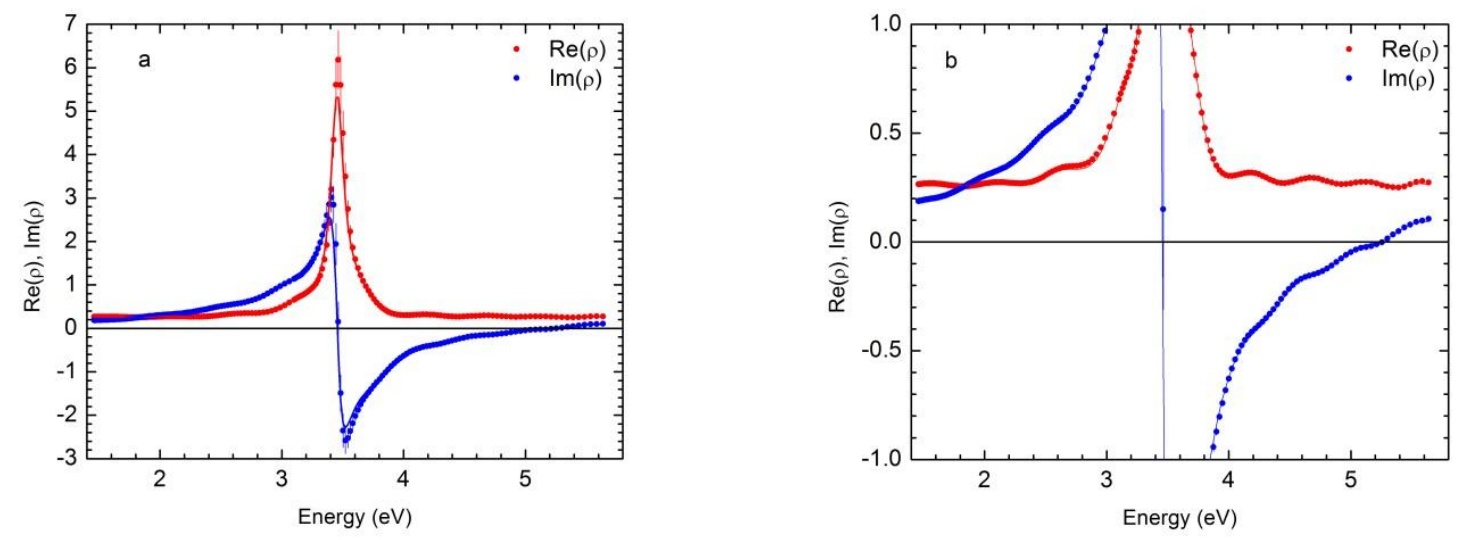

Figure 2. The real and imaginary parts of $\rho$ and the errors determined using the 2-MGE. The right plot (b) is the same as the left plot (a) but with an expanded $y$-axis. The experimental data are represented with dots, the errors by vertical lines extending above and below the data points, and the fit by the solid line. 


\section{Ellipsometry Data Modeling}

The fitting of the data was done using the procedure described in refs. [5-7], where the figure of merit was the traditional $\chi^{2}$, given by

$$
\chi^{2}=\frac{1}{N-m} \sum_{i=0}^{N}\left[\frac{\left(\rho_{R e, d}\left(\lambda_{i}\right)-\rho_{R e, c}\left(\lambda_{i}, K\right)\right)^{2}}{\delta \rho_{R e, d}\left(\lambda_{i}\right)^{2}}+\frac{\left(\rho_{I m, d}\left(\lambda_{i}\right)-\rho_{I m, c}\left(\lambda_{i}, K\right)\right)^{2}}{\delta \rho_{I m, d}\left(\lambda_{i}\right)^{2}}\right]
$$

The subscript Re and Im represent the real and imaginary parts of $\rho$, and $d$ and c represent the data and calculated values of $\rho$, respectively. The quantities $\delta \rho_{\text {Re,d }}\left(\lambda_{\mathrm{i}}\right)$ and $\delta \rho_{\mathrm{Im}, \mathrm{d}}\left(\lambda_{\mathrm{i}}\right)$ represent the error of the real and imaginary parts of the $\mathrm{i}^{\text {th }}$ data point and the $\mathrm{m}$-dimensional vector $\mathbf{K}$ represents the fitted quantities. The value of $\chi^{2}$ can be used as a figure of merit of the fit: If it is close to 1 , then the model fits the data, but if it is considerably larger than 1 , then the model does not fit the data. This criterion is only strictly valid for linear systems where the errors are entirely stochastic. Unfortunately, this is not the case for ellipsometry measurements: The models required to fit ellipsometry data are decidedly non-linear, and the errors in the data contain both stochastic and systematic components. Since there is no other reasonable alternative, the $\chi^{2}$ near 1 criterion can still be used, but one must be aware that this only an approximate criterion.

The fit to the data for the substrate was performed using a two-layer model, where the refractive index of each layer was modeled using the Sellmeier parameterization:

$$
\varepsilon(\lambda)=n(\lambda)^{2}=1+\frac{A \lambda^{2}}{\lambda^{2}-\lambda o^{2}}
$$


The quantity $\varepsilon$ is the dielectric function, $n$ is the refractive index, $\lambda$ is the wavelength (in $\mathrm{nm}$ ), $A$ is the amplitude and $\lambda_{\mathrm{o}}$ is the resonance wavelength (considerably lower than any measured wavelength). The $\varepsilon$ of the Sellmeier approximation is real, indicating that there is no significant absorption. The resulting fit to the overlayer/glass substrate starting material resulted in $\chi^{2}=0.93$, where the overlayer thickness was $329 \pm 7 \mathrm{~nm}$, the Sellmeier parameters of the overlayer were $A_{o}=$ $1.164 \pm 0.005, \lambda_{0,0}=86 \pm 3 \mathrm{~nm}$, and the Sellmeier parameters of the glass substrate were $A_{s}=1.170 \pm 0.006$, $\lambda_{s, 0}=80 \pm 4 \mathrm{~nm}$. At $550 \mathrm{~nm}$, the refractive indices are $\mathrm{n}_{\mathrm{o}}=1.481$ and $\mathrm{n}_{\mathrm{s}}=1.482$. The refractive index of fused silica is 1.460 at this wavelength.

In fitting the data to the nanostructured porous film, it was immediately clear that a simple film on substrate model would not work: The resulting calculated $\rho$ near the central resonance was much narrower than the data, and it did not fit the oscillations in the wings at all. The oscillations in the wings indicate that the model must include a rather thick layer between the film and the substrate. Furthermore, the structure of the film is extremely complicated, and the model must contain transition layers between the ambient and the film, and between the film and the next layer. Finally, one cannot use published or measured data for the substrate alone, since gradual changes in the substrate refractive index will be seen in an ellipsometry experiment as the refractive index at the top of the substrate [5].

The final model used to fit the spectrum in Fig. 1 consisted in 5 layers, given below:

0. Ambient

1. Transition layer, modelled using a two component Bruggeman effective medium approximation (BEMA) where the fraction of the two components varies with depth into the film. 
2. Film, modeled using the Sellmeier parameterization with a distribution of the amplitude. This is the central thickest part of the film stack.

3. Transition layer, similar to layer 1.

4. Thick layer, modeled using the Sellmeier parameterization.

5. Substrate, modeled using the Sellmeier parameterization.

Since there is no significant absorption in the film structure, the Sellmeier parameterization (Eq. 3) was used to model the wavelength dispersion of all the layers.

The transition layers ( 1 and 3 ) are modeled using the two component Bruggeman effective medium approximation (BEMA) [8], where the dielectric function $\varepsilon_{\mathrm{h}}$ is given by

$$
0=f \frac{\varepsilon_{1}-\varepsilon_{h}}{\varepsilon_{1}+2 \varepsilon_{h}}+(1-f) \frac{\varepsilon_{2}-\varepsilon_{h}}{\varepsilon_{2}+2 \varepsilon_{h}}
$$

and $f$ is the fraction of component 1 . For these transition layers, the fraction $f$ depends on the depth into the transition layer and the dielectric functions $\varepsilon_{1}$ and $\varepsilon_{2}$ are given by the dielectric functions of the layer above and below the transition layer, respectively.

To model the fraction $f$ of the transition layers, we have used the normalized incomplete beta function, often found in statistics [9], which is given by

$$
I_{x}(a, b)=\frac{B_{x}(a, b)}{B(a, b)}=\frac{1}{B(a, b)} \int_{n}^{x} t^{a-1}(1-t)^{b-1} d t
$$

The quantity $B(a, b)$ is the complete beta function and is just the integral portion of the expression where $x=1$. The values of $a$ and $b$ must be greater than 0 . This function has a 
domain $(x)$ of 0 to 1 and a range of 0 to 1 . Figure 3 shows the plots of the incomplete beta function for several different values of $a$ and $b$. If the domain $x$ is scaled to the thickness of the transition layer, then $I_{x}(a, b)$ represents the fraction to be used in the expression for the BEMA. If $a=b=1$, then the fraction will go from 0 to 1 linearly with the value of $x$. If $a=b<1$, then the fraction profile will be relatively flat near $x=0.5$, and the profile will be flatter as $a=b$ becomes closer to zero. If $a=b>1$, the fraction profile looks like a rounded step function with $f=0.5$ at $x=0.5$. If $a$ and $b$ are not equal, then the $f=0.5$ point will occur at a different value of $x$. As can be seen, many realistic transition layers can be expressed using this function which needs only two fitting parameters. Using this function, the complete transition layer can be modeled using only three parameters: $a, b$, and the total layer thickness.

The complete Abeles matrices for the transition layer are then calculated using 20 sublayers where the dielectric functions are determined by the BEMA and the fractions using the incomplete beta function. To speed up the calculation and improve the accuracy, the sublayers are

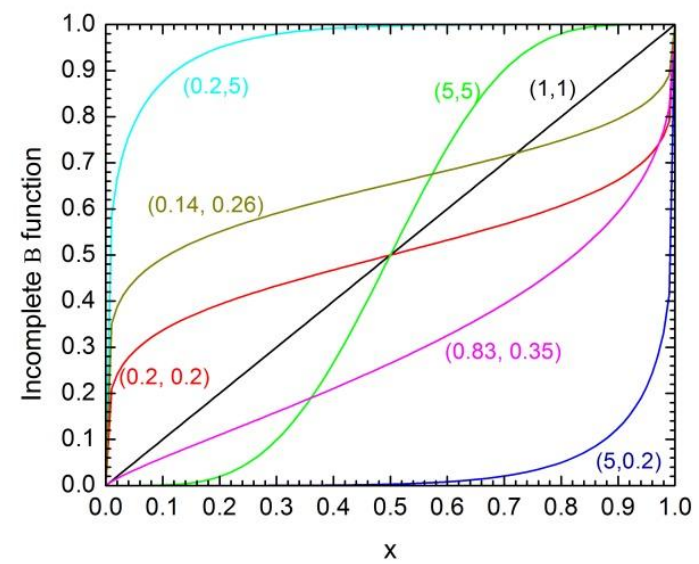

Figure 3. The incomplete beta function plotted as a function of its domain $\mathrm{x}$ for several values of $(a, b)$.

not treated as a layer with a constant dielectric function, but rather are modeled as a linearly graded profile where the dielectric function of the bottom of the $n^{\text {th }}$ sub layer is equal to the dielectric function of the top of the $(n+1)^{\text {th }}$ sublayer. The Abeles matrices for each sublayer are 
then calculated using the expressions given in references 10 and 11 and summarized in reference 5 .

As can be seen from Fig. 1, the film itself is quite nonuniform on the microscopic scale. That is, the optical functions of the film might vary considerably over the spot sampled using the 2MGE (an ellipse 3X5 mm). To take this into account, the amplitude of the Sellmeier parameterization $A$ was convoluted using a Gaussian distribution.

$$
p(A)=\frac{1}{\sigma \sqrt{2 \pi}} \exp \left(\frac{-\left(A-A_{0}\right)^{2}}{2 \sigma^{2}}\right)
$$

Where $A_{\mathrm{o}}$ is the central value of the distribution and the width of the distribution is given by $\sigma$.

This is similar to the procedure described in ref. 12 .
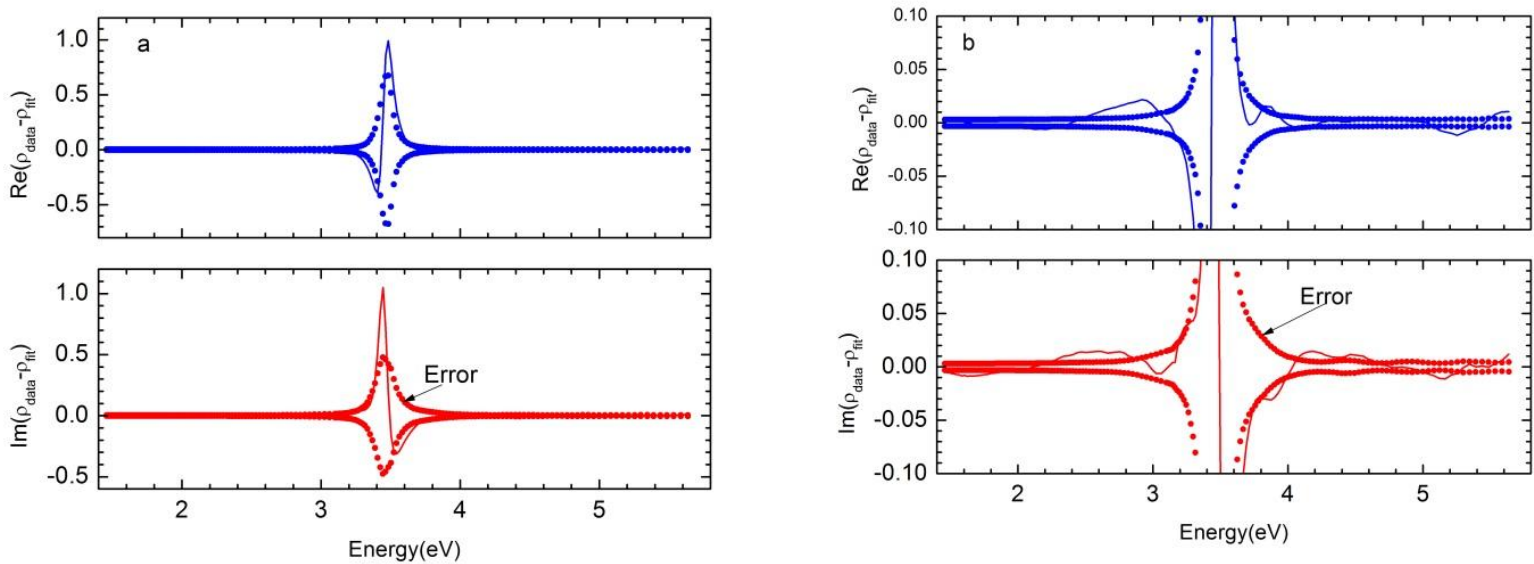

Figure 4. The $\rho$ data minus the $\rho$ fit for the real (top) and imaginary (bottom) parts. For comparison, dots have been added to represent the experimental errors, placed above and below the zero line. The right plot (b) is the same as the left plot (a) but with an expanded y-axis. 
The best fit to the data employed 15 fitted parameters resulting in a final $\chi 2=2.87$. The parameters, their values, and the correlated error are shown in Table I and the resulting $\rho$ is shown in Figure 2 and the data minus the fit is shown in Figure 4. The normalized cross correlation coefficients were also calculated, and several parameters were identified as being pair-wise correlated. That is, the two parameters were correlated with each other (with a normalized cross correlation coefficient $>0.9$ ), but otherwise not significantly correlated with any of the other fitted parameters. The high correlation coefficients also result in an increase in the correlated error. Seven of the parameters are not significantly correlated with any of the other parameters, and are indicated by " $\mathrm{nc}$ " in the Correlations column. 
Table I. The final fitted parameters and their errors for the fit shown in Figure 1. The errors shown are the correlated errors. 8 parameters are pair-wise correlated with a normalized cross correlation coefficient $>0.9$, as indicated in the "Correlations" column by the letters $a, b, c$, and d. These 8 parameters are not correlated with the other parameters. The uncorrelated parameters are indicated by "nc".

$\begin{array}{ll}\text { Layer \# } & \text { Parameter } \\ 1 & \text { Thickness }(\mathrm{nm}) \\ 1 & \text { Transition layer Incomplete beta } a \\ 1 & \text { Transition layer Incomplete beta } b \\ 2 & \text { Thickness ( } \mathrm{nm} \text { ) } \\ 2 & \text { Sellmeier Amplitude } A \\ 2 & \text { Sellmeier Amplitude Sigma } \sigma \\ 2 & \text { Sellmeier Resonance } \lambda_{0} \text { (nm) } \\ 3 & \text { Thickness (nm) } \\ 3 & \text { Transition layer Incomplete beta } a \\ 3 & \text { Transition layer Incomplete beta } b \\ 4 & \text { Thickness (nm) } \\ 4 & \text { Sellmeier Amplitude } A \\ 4 & \text { Sellmeier Resonance } \lambda \circ(\mathrm{nm}) \\ 5 & \text { Sellmeier Amplitude } A \\ 5 & \text { Sellmeier Resonance } \lambda_{0} \text { (nm) }\end{array}$

Value and corr. error Correlations

$66.8 \pm 2.5 \quad n c$

$0.826 \pm 0.316$

$0.353 \pm 0.121 \quad a$

$93.8 \pm 1.5 \quad$ nc

$0.305 \pm 0.007 \quad n c$

$0.034 \pm 0.003 \quad n c$

$125 \pm 6 \quad n c$

$40.3 \pm 1.7 \quad n c$

$0.144 \pm 0.093 \quad b$

$0.265 \pm 0.158 \quad b$

$982 \pm 7 \quad$ nc

$1.144 \pm 0.013 \quad$ c

$76 \pm 4 \quad d$

$1.123 \pm 0.013 \quad$ c

$77 \pm 4 \quad d$ 


\section{Discussion}

The refractive index profile for this sample depends significantly on the depth into the film and is shown in Fig. 5 at $\lambda=550 \mathrm{~nm}$. The refractive index of the first transition layer increases from the ambient to the refractive index of layer 2 at a thickness of $66.8 \mathrm{~nm}$. Here, the average refractive index is 1.15 but can vary $\pm 0.015(1 \sigma)$ over the spot

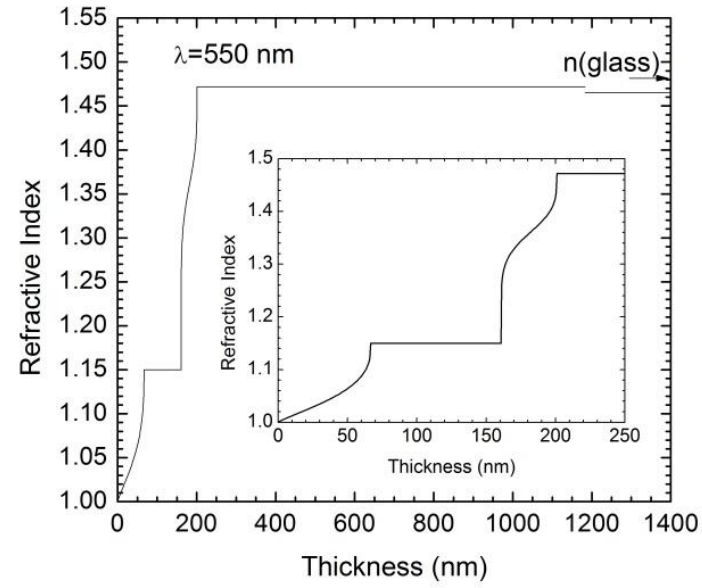

Figure 5. The refractive index profile of the nanostructured film at $550 \mathrm{~nm}$. The insert shows the refractive index of the near-surface region on an expanded scale.

examined by the 2-MGE. The refractive index of the second transition layer (i.e., layer 3 )

increased from the refractive index of layer 2 to the refractive index of layer 4 , but the profile shows that the average refractive index stays in the range of $\sim 1.3-1.4$. The refractive index of layer 4 is $1.472 \pm 0.004$, while the substrate refractive index is $1.465 \pm 0.004$, compared to the refractive index of the substrate before film deposition of 1.482 . 

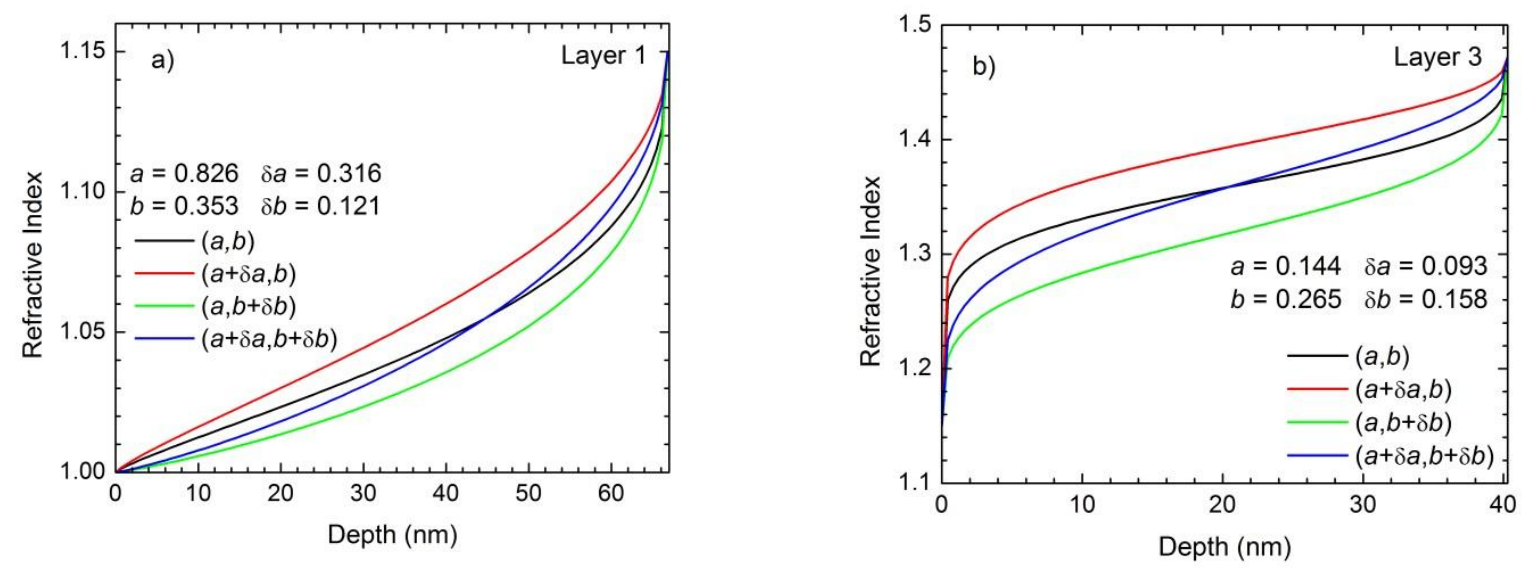

Figure 6. Refractive index profiles at $\lambda=550 \mathrm{~nm}$ for the graded layers 1 and 3 . The $a$ and $b$ and the associated errors $\delta a$ and $\delta b$ are the fitted parameters and correlated errors, given in Table I.

As mentioned above, some of the fitted parameters are pair-wise correlated. The strongest correlations are between the $a$ and $b$ parameters for the incomplete Beta functions describing the graded layer profiles of Layers 1 and 3. Figure 6 shows a plot of the depth-dependent refractive index for layers 1 and 3 using different values of the $a$ and $b$ parameter for the incomplete Beta function. Examining Layer 1 , it can be seen that $a$ and $b$ are primarily anticorrelated, in that if both $a$ and $b$ are increased by $\delta a$ and $\delta b$ respectively, the profiles are nearly the same; a similar conclusion can be made concerning layer 3 . If $a$ is increased while keeping $b$ constant, then the profile increases, while increasing $b$ while keeping a constant results in a decrease in the profile. These plots emphasize that there is a significant error associated with the depth-dependent refractive index of the two transition layers 1 and 3. However, it is clear that the fit does indicate that the refractive index of layer 1 rises slowly from the ambient, and the refractive index of layer 3 has a near-saddle point in the middle of the layer. The error in the refractive index of layer $2\left(n_{2}=1.150 \pm 0.015\right)$ is not included in Figure 5 , but clearly it leads to an uncertainty of the end of layer 1 and the beginning of layer 3 . If this 
uncertainty were included, then the sharp transitions between layers 1 and 2 and between 2 and 3 would not be as abrupt as shown in Fig. 5 . The uncertainty in the refractive index of layer 4 is also not included, but is rather small $\left(n_{4}=1.472 \pm 0.004\right)$.

The slow increase in the surface refractive index can be understood from the surface profile shown in the scanning transmission electron microscope (STEM) image in Figure 1. Near the top of the surface, the film consists of mostly voids and some of the nanostructured film, which also has a significant fraction of voids. The gradual increase in glass fraction with depth into the film is clearly observable in Fig. 1 and is modeled well by the incomplete Beta function resulting in the refractive index profile as shown in Fig. 6 . Layer 3 is shown as a transition layer right below the nanotextured film and above the much thicker borosilicate film. This conclusion is valid even with the uncertainty of the profile as shown in Fig. 6 . Comparing to the STEM image of Fig. 1, it is clear that this transition layer is the typical rough interface layer encountered in many thin film ellipsometric measurements, where the interface depth varies with position. At a depth of $1183 \mathrm{~nm}$, another transition is observed, where there is a small difference in index of refraction between layers 4 and 5 (1.472 versus 1.465$)$. Layer 4 is clearly the borosilicate glass layer that has been spinodally phase separated due to the heat treatment, but has not been etched. Therefore, a small difference in refractive index is to be expected. It should be noted that the ellipsometric thickness of $982 \mathrm{~nm}$ is different from the thickness ( 1180 $\mathrm{nm}$ ) observed by STEM; this is probably due to the specimen for the STEM measurements being prepared from a different position than that used for the ellipsometry measurements. The observation that the refractive index of layer 5 is somewhat smaller than that measured for the uncoated glass indicates that the glass has undergone 
structural/chemical modifications to lower its near-surface refractive index. This could result from either a leaching of $\mathrm{NaO}_{2}-\mathrm{B}_{2} \mathrm{O}_{3}$ material and/or an increase in the microvoid fraction, both of which would lower the index of refraction.

\section{Conclusion}

We have shown that a sophisticated analysis of the ellipsometry spectrum from a very complicated nanoporous glass film results in good fit to the data with $\chi^{2}=2.87$. The fit involves 15 parameters describing the thicknesses and optical properties of 5 layers. While this is a large number of parameters, 7 show very little cross correlation, and the 8 correlated parameters are only pair-wise correlated. That is, each of the 4 pairs of correlated parameters are correlated with each other, but not significantly correlated with the other 13 parameters.

Layers 1 and 3 are treated as transition layers with a refractive index that varies with depth. The refractive index is calculated using the two-component Bruggeman effective medium approximation [8], where the component spectroscopic refractive indices are taken from the material above and below the respective layer. The fraction of the first component is parameterized using the incomplete Beta function, which has two parameters ( $a$ and $b$, both $>1$ ) and a domain and range of 0 to 1 . Thus, the transition layers could be parameterized using only 3 parameters: $a, b$, and thickness.

Layer 2 was modeled using the Sellmeier equation (Eq. 3), but where the Sellmeier amplitude was distributed using a Gaussian distribution. This was done to simulate the fact that the light spot from the ellipsometer samples an area $\sim 3 \times 5 \mathrm{~mm}$, which will contain a large 
variation in film refractive indices. Layers 4 and 5 were modeled using the simple Sellmeier equation.

This work emphasizes the necessity of using proper statistics in the analysis of ellipsometry data, particularly when the surface structure being examined is complicated. Not only must a figure of merit be used to determine whether or not the model actually fits the structure being measured, but the correlated errors and cross-correlation coefficients must be calculated to indicate possible interrelationships between the fitted parameters.

\section{Acknowledgements}

This work was in part supported by the Laboratory Directed Tip Innovation Program of Oak

Ridge National Laboratory, managed by UT-Battelle, LLC for the U.S. Department of Energy. STEM research was supported by the U.S. Department of Energy (DOE), Office of Science, Basic Energy Sciences (BES), Materials Sciences and Engineering Division. 


\section{References}

1. T. Aytug, J. T. Simpson, A. R. Lupini, R. M. Trejo, G. E. Jellison, I. N. Ivanov, S. J.

Pennycook, D. A. Hilesheim, K. O. Winter, D. K. Christen, S. R. Hunter, and J. A. Haynes, Optically transparent, mechanically durable, nanostructured superhydrophobic surfaces enabled by spinodally phase-separated glass thin films, Nanotechnol. 24 (2013) 315602.

2. T. Aytug, A. R. Lupini, G. E. Jellison, P. C. Joshi, I. H. Ivanov, T. Liu, P. Wang, R. Menon, R. M. Trejo, E Lara-Curzio, S. R. Hunter, J. T. Simpson, M. P, Paranthaman and D. K. Christen, Monolithic graded-refractive-index glass-based antireflective coatings: broadband/omnidirectional light harvesting and self-cleaning characteristics, J. Mat. Chem. C (2015) 5440-5449.

3. G. E. Jellison, Jr. and F. A. Modine, Two-modulator generalized ellipsometry: experiment and calibration, Appl. Opt. 36 (1997) 8184-8189.

4. G. E. Jellison, Jr. and F. A. Modine, Two-modulator generalized ellipsometry: theory, Appl. Opt. 36 (1997) 8190-8198.

5. G. E. Jellison, Jr., Data analysis for spectroscopic ellipsometry, Thin Solid Films 234 (1993) 416-422.

6. G. E. Jellison, Jr., Spectroscopic ellipsometry data analysis: measured versus calculated quantities, Thin Solid Films 313-314 (1998) 33-39.

7. G. E. Jellison, Jr., Data analysis for spectroscopic ellipsometry, in H. G. Tompkins and E. A. Irene (eds.) Handbook of Ellipsometry, William Andrew, Springer, Norwich, NY USA (2005), Ch. 3. 
8. D. A. G. Bruggeman, Ann. Phys., (Leipzig) 24 Berechnung verschiedener physikalisher konstanten vor heterogenen substanzen (Calculation of different physical constants of heterogeneous substances), (1935) 636.

9. W. H. Press, S. A. Teukolsky, W. T. Vettering, and B. P. Flannery, Numerical Recipes, Cambridge University Press, $3^{\text {rd }}$ edition, (2007) p. 270.

10. F. Abeles, Optical properties of inhomogeneous films, in E. Passaglia, R. R. Stromberg and J. Kruger (eds.) Ellipsometry in the Measurement of surfaces and thin films, Natl. Bur. Std. Misc. Publ. 256 US Government Print Office, Washington, DC (1964) pp. 41-56.

11. R. Jacobsson, Light reflection from films of continuously varying refractive index, in $E$. Wolf (ed.), Progress in Optics, 5, Wiley, (1965), pp. 247-286.

12. G. E. Jellison, Jr. and J. W. McCamy, Sample depolarization effects from thin films of ZnS on GaAs as measured by spectroscopic ellipsometry, Appl. Phys. Lett. 61 (1992) 512. 


\section{Figure Captions}

Figure 1. 'Z-contrast' cross-sectional scanning transmission electron microscopy (STEM) image of the glass coating. The fiduciary mark represents $500 \mathrm{~nm}$. The layers marked 1, 2, and 3 represent the transition layer between air and the film, the film, and the transition layer between the film and the unetched borosilicate glass film.

Figure 2. The real and imaginary parts of $\rho$ and the errors determined using the 2-MGE. The right plot (b) is the same as the left plot (a) but with an expanded $y$-axis. The experimental data are represented with dots, the errors by vertical lines extending above and below the data points, and the fit by the solid line.

Figure 3. The incomplete beta function plotted as a function of its domain $\mathrm{x}$ for several values of $(a, b)$.

Figure 4. The $\rho$ data minus the $\rho$ fit for the real (top) and imaginary (bottom) parts. For comparison, dots have been added to represent the experimental errors, placed above and below the zero line. The right plot (b) is the same as the left plot (a) but with an expanded $y$-axis.

Figure 5. The refractive index profile of the nanostructured film at $550 \mathrm{~nm}$. The insert shows the refractive index of the near-surface region on an expanded scale.

Figure 6 . Refractive index profiles at $\lambda=550 \mathrm{~nm}$ for the graded layers 1 and 3 . The $a$ and $b$ and the associated errors $\delta a$ and $\delta b$ are the fitted parameters and correlated errors, given in Table $I$. 


\section{Table Caption}

Table I. The final fitted parameters and their errors for the fit shown in Figure 1 . The errors shown are the correlated errors. 8 parameters are pair-wise correlated with a normalized cross correlation coefficient $>0.9$, as indicated in the "Correlations" column by the letters $a, b, c$, and d. These 8 parameters are not correlated with the other parameters. The uncorrelated parameters are indicated by "nc". 